\title{
NOTES
}

\section{Optical Absorbency Study on the Hydration of Polyvinylpyrrolidone Films Containing Cobalt Chloride}

\author{
Soichi OtsuKI and Kimihiro ADACHI \\ Government Industrial Research Institute, Osaka \\ 1-8-31, Midorigaoka, Ikeda 563, Japan
}

(Received December 18, 1992)

\begin{abstract}
KEY WORDS Optical Humidity Detection / Absorption Spectrum / Cobalt Chloride / Polyvinylpyrrolidone / N-Methyl-2-Pyrrolidone / Water Vapor Sorption /
\end{abstract}

Polymer films are used as sensing membranes of sensors for various chemical species in gas and liquid. Recently sensing devices using an optical fiber have drawn considerable attention because of advantages over electrochemical ones. ${ }^{1,2}$ Cobalt (II) chloride $\left(\mathrm{CoCl}_{2}\right)$ entrapped in polymer films has been studied for optical moisture detection by many workers. ${ }^{3-7}$ In spite of these reports, fundamental knowledge on spectroscopic and water sorptive properties is insufficient.

The work described here is part of an ongoing investigation into the possible applications of optical methods for quantitative moisture sensor system. In a previous study, ${ }^{8}$ we examined the relative humidity $(R H)$ dependence of the absorption spectrum and water vapor sorption of polyvinylpyrrolidone (PVP) films containing $\mathrm{CoCl}_{2}$. It was concluded that, when $R H$ increases, the 4-coordinate form of a cobalt ion changes to the 6-coordinate one and the amide group of the polymer coordinating to the cobalt ion is replaced by a water molecule. In the above study, however, the result is qualitative due to a single beam system used in the measurement of absorption spectrum. In this report, the relation between the absorbance of films and ambient humidity was investigated quantitatively.
Changes in absorption spectrum of the PVP films with $R H$ were compared with the absorption spectra of $\mathrm{N}$-methyl-2-pyrrolidone solutions of $\mathrm{CoCl}_{2}$, which were measured at various water concentrations. The absorbance$R H$ curves of the film are discussed in relation to the coordinate state of $\mathrm{CoCl}_{2}$ in the polymer.

\section{EXPERIMENTAL}

\section{Sample Preparation}

Polyvinylpyrrolidone (PVP) (MW 360000, Nacalai Tesque), $N$-methyl-2-pyrrolidone (MP) (guaranteed reagent, Ishizu Pharmaceutical), and $\mathrm{CoCl}_{2} \cdot 6 \mathrm{H}_{2} \mathrm{O}$ (guaranteed reagent, Kishida Chemical) were used as received. Aqueous solutions were prepared with $3.8 \%$ $(\mathrm{g} / 100 \mathrm{ml}$ solution) of PVP and various concentrations of $\mathrm{CoCl}_{2}$ at $0,0.167,0.333$, and $0.667 \%$ (g/100 ml solution). $0.2 \mathrm{ml}$ of each solution was cast on a $36 \times 13 \mathrm{~mm}$ glass plate to produce moisture sensitive films containing $\mathrm{CoCl}_{2}$ at various concentrations of $0,0.33$, 0.67 , and $1.35 \mathrm{~mol} \mathrm{~kg}^{-1}$ ( $\left.\mathrm{mol} \mathrm{kg}^{-1} \mathrm{PVP}\right)$, respectively. Film thickness was about $16 \mu \mathrm{m}$, as calculated from the volume of the coated solution by assuming that the density of all films is equal to 1.0 . 
Anhydrous $\mathrm{CoCl}_{2}$ was prepared by drying $\mathrm{CoCl}_{2} \cdot 6 \mathrm{H}_{2} \mathrm{O}$ at $160^{\circ} \mathrm{C}$ under vacuum and dissolved in MP at concentrations of $0,0.33$, 0.67 , and $1.35 \mathrm{~mol} \mathrm{~kg}^{-1}\left(\mathrm{~mol} \mathrm{~kg}^{-1} \mathrm{MP}\right)$.

Measurement of the Absorption Spectra of PVP Films

A gas flow system was constructed by use of a Shin-ei SRH-1R humidity generator. The absorption spectrum of the sample was measured using a JASCO UVDEC-1 spectrometer under the condition of desired relative humidity $(R H)$. The measurements were carried out at $20.0 \pm 0.2^{\circ} \mathrm{C}$. Detail procedures can be found in the preceding paper. ${ }^{9}$

\section{Measurement of the Absorption Spectrum for MP Solutions}

The water concentration in the PVP film was determined previously ${ }^{8}$ when the film was equilibrated in air streams of various $R H$ s. To a measured volume of the MP solution of $\mathrm{CoCl}_{2}$ was added water so that the water concentration became the same as that in the PVP film. For example, water concentrations used for the MP solution containing 1.35 mol kg-1 of $\mathrm{CoCl}_{2}$ were $66.7,41.2,16.3,11.1$, 6.2 , and $0 \mathrm{~mol} \mathrm{~kg}^{-1}\left(\mathrm{~mol} \mathrm{~kg}^{-1} \mathrm{MP}\right)$, which are the same as the water concentrations in the PVP film at $91,81,62,42,22$, and $0 \% R H$, respectively, in the desiccation process. The absorption spectrum of the MP solutions was measured using a Shimazu UV-2100S spectrometer and a remountable flow-through cell with a path length of $25 \mu \mathrm{m}$.

\section{RESULTS AND DISCUSSION}

Humidity Dependence of Absorption Spectrum

Absorption spectra of a PVP film containing $1.35 \mathrm{~mol} \mathrm{~kg}^{-1}$ of $\mathrm{CoCl}_{2}$ were measured starting at $0 \% R H$ and ending at $90 \% R H$. The results are shown in the range from 450 to $750 \mathrm{~nm}$ in Figure 1. Overall decrease in absorbance is observed in the range from about 550 to $720 \mathrm{~nm}$ with increasing $R H$. In addition, it can be seen

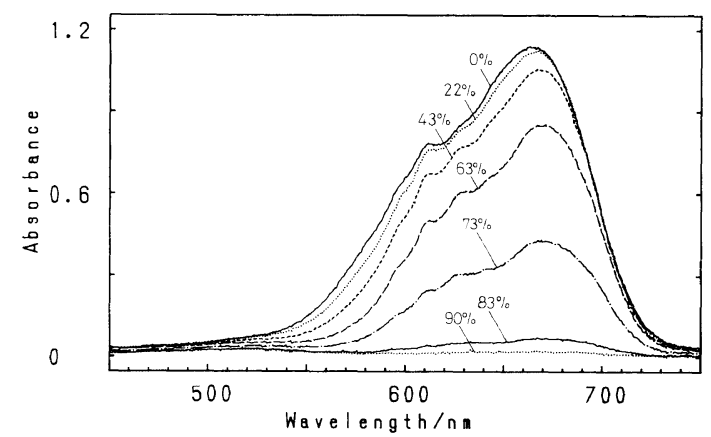

Figure 1. Absorption spectra of a PVP film containing $1.35 \mathrm{~mol} \mathrm{~kg}^{-1} \mathrm{CoCl}_{2}$ at a varying $R H$.

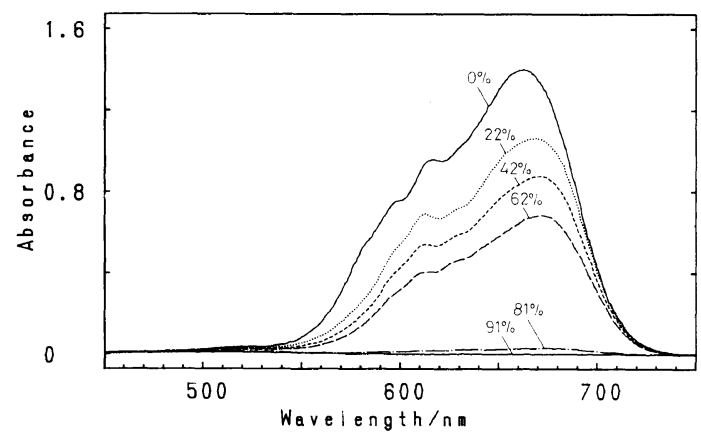

Figure 2. Absorption spectra of an MP solution containing $1.35 \mathrm{~mol} \mathrm{~kg}^{-1} \mathrm{CoCl}_{2}$ at varying $R H \mathrm{~s}$, which correspond to the water concentration used in the measurement.

that the absorption maximum shifts from 666 $\mathrm{nm}$ at $0 \% R H$ to $673 \mathrm{~nm}$ at $71 \% R H$. Similar spectral features were also observed for both PVP films containing 0.33 and $0.67 \mathrm{~mol} \mathrm{~kg}^{-1}$ of $\mathrm{CoCl}_{2}$.

Changes in absorption spectrum of the PVP film with $R H$ were compared with the absorption spectra of MP solutions of $\mathrm{CoCl}_{2}$ mixed with a varying amount of water. The spectral change for the MP solution of $1.35 \mathrm{~mol} \mathrm{~kg}^{-1} \mathrm{CoCl}_{2}$ is shown in Figure 2. The absorbance decreases as a whole in the range from about 550 to $720 \mathrm{~nm}$ with increasing water concentration from 0 to $66.7 \mathrm{~mol} \mathrm{~kg}^{-1}$, which corresponds to the increase of $\mathrm{RH}$ from 0 to $91 \%$. This is apparently the result of alteration of cobalt ( $\mathrm{Co}$ ) complexes from the 4coordinate to the 6-coordinate. It has been 
demonstrated that $\mathrm{MP}^{10}$ and $\mathrm{PVP}^{11}$ form complexes with transition metal ions such as $\mathrm{Co}^{2+}$ in which the metal atom is coordinatively bonded to the carbonyl oxygen atom of the pyrrolidone residue. Therefore, it is expected that this type of coordination bond exists herein in MP solutions and PVP films and participates in the change of Co complexes from the 4-coordinate to the 6-coordinate.

A longer-wavelength shift of the absorption maximum of about $665 \mathrm{~nm}$ is also observed in analogy with the absorption spectrum of PVP films. The absorption maximum shifts from 661.6 to $671 \mathrm{~nm}$ for the MP solution of 1.35 mol kg-1 of $\mathrm{CoCl}_{2}$ when the water concentration is increased from 0 to $16.3 \mathrm{~mol} \mathrm{~kg}^{-1}$, which corresponds to an $R H$ change from 0 to $62 \%$ for the PVP film. Shifts of the absorption maximum were $660.3 \rightarrow 671 \mathrm{~nm}$ and $660.8 \rightarrow 671 \mathrm{~nm}$, respectively, for the MP solutions of 0.33 and $0.67 \mathrm{~mol} \mathrm{~kg}^{-1}$ of $\mathrm{CoCl}_{2}$ when the same increase of the water concentration was achieved. It seems that these shifts of the absorption maximum are the result of change in relative intensity of two different maxima; the absorption maximum of about $665 \mathrm{~nm}$ is supposed to consist of the two maxima located at about 660 and $670 \mathrm{~nm}$. These maxima may be attributed to $\mathrm{CoX}_{2} \mathrm{Cl}_{2}$ and $\mathrm{CoXCl}_{3}{ }^{-}$, respectively, where $\mathrm{X}$ is the carbonyl group of MP. It has been demonstrated that the addition of water to an acetone solution of $\mathrm{CoCl}_{2}$ makes the absorption spectrum resemble that of $\mathrm{CoCl}_{3}{ }^{-} \cdot{ }^{12} \mathrm{~A}$ similar effect of addition of water is expected to occur in MP solutions. It seems that water molecules knock chloride ions away from a $\mathrm{Co}$ complex; the chloride ion attacks another complex and eliminates a carbonyl group of MP, generating $\mathrm{CoXCl}_{3}{ }^{-}$.

In addition, the absorption maxima at the water concentration of $0 \mathrm{~mol} \mathrm{~kg}^{-1}$ are 660.3 , 660.8 , and $661.6 \mathrm{~nm}$, respectively, for MP solutions of $0.33,0.67$, and $1.35 \mathrm{~mol} \mathrm{~kg}^{-1}$ of $\mathrm{CoCl}_{2}$. Thus, when a higher concentration of $\mathrm{CoCl}_{2}$ is used, this maximum becomes longer, indicating an increase in relative intensity of the maximum of about $670 \mathrm{~nm}$. It seems that a higher concentration of $\mathrm{CoCl}_{2}$ produces binuclear complexes held together by chloride ions, which have the structure

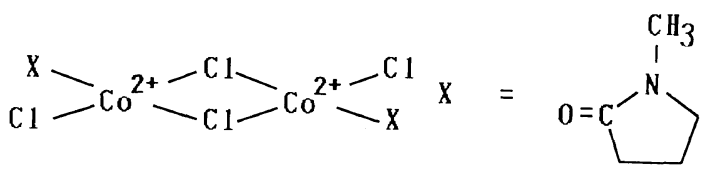

Although the concentration of $\mathrm{CoCl}_{2}, 1.35$ mol kg-1, is smaller than the corresponding concentration of MP in the solution, 10.1 mol kg-1, this concentration seems sufficient to form binuclear complexes. Since each Co ion of the binuclear complexes forms three bonds to chlorine atoms in a similar manner to $\mathrm{CoXCl}_{3}{ }^{-}$, these complexes are considered to have an absorption maximum at the same wavelength as $\mathrm{CoXCl}_{3}{ }^{-}$. Therefore, the relative intensity of the maximum at about $670 \mathrm{~nm}$ is larger at a higher concentration of $\mathrm{CoCl}_{2}$, where more binuclear complexes exist in the solution. Little difference in the shift of the absorption maximum was observed between PVP films containing different concentrations of $\mathrm{CoCl}_{2}$, but a small amount of binuclear complexes is probably formed also in dry films.

\section{Absorbance vs. RH Curve}

The $R H$ dependence of the absorbance at $670 \mathrm{~nm}$ of the PVP- $\mathrm{CoCl}_{2}$ films is shown in Figure 3 . The absorbance decreases slowly with increasing $R H$ in the range from 0 to $63 \% R H$ and drops steeply in the range from 63 to $83 \% R H$. Figure 4 shows the absorbance at $670 \mathrm{~nm}$ of the MP solutions as a function of $R H$ corresponding to the water concentration used in the measurement. These curves of absorbance vs. $R H$ for the MP solutions should be compared with those in the desiccation for PVP films, depicted in Figure 3, because the water concentrations of the MP solutions were determined to be the same as those in the desiccation of the PVP film. It is found by this 


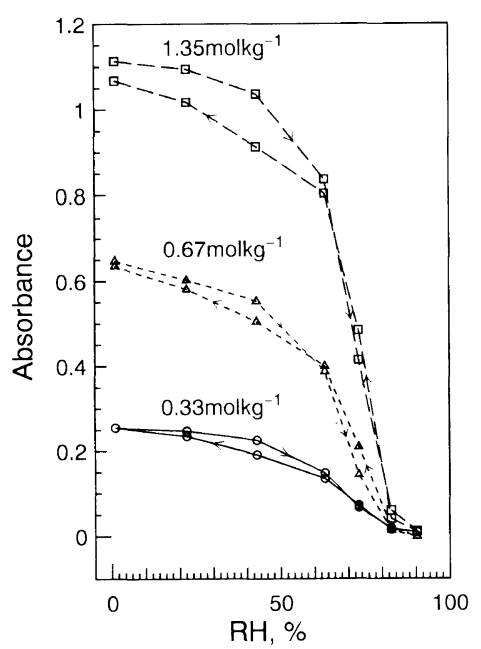

Figure 3. Absorbance at $670 \mathrm{~nm} v s$. $R H$ curves for PVP films containing various concentrations of $\mathrm{CoCl}_{2}$.

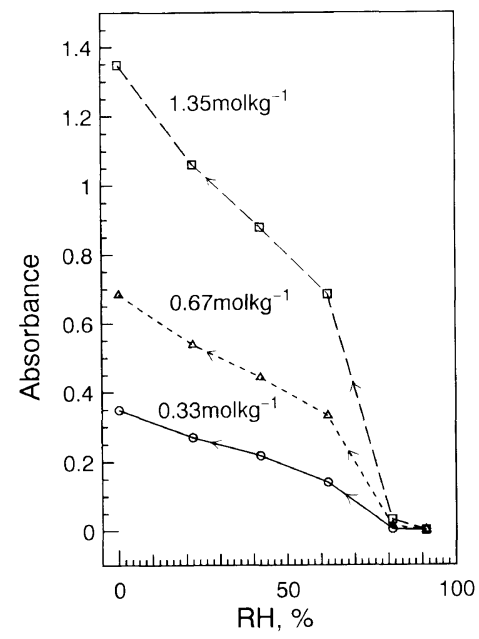

Figure 4. Absorbance at $670 \mathrm{~nm} v s$. $R H$ curves for MP solutions containing various concentrations of $\mathrm{CoCl}_{2} . \mathrm{RH}$ corresponds to the water concentration used in the measurement.

comparison that the absorbance less steeply decreases with increasing $R H$ in the low- and middle- $R H$ regions for the PVP films than the case of MP solutions. This indicates that Co complexes in the film interact with water less easily than those in solution at the same concentration of water. It seems that the water molecules adsorbed in the film in the low- and

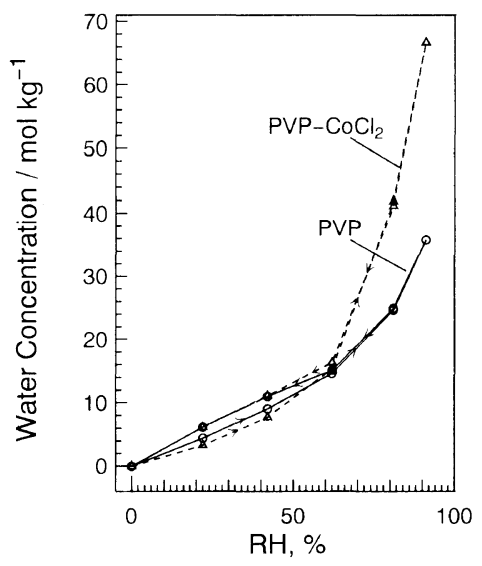

Figure 5. Water concentration in PVP films with and without $\mathrm{CoCl}_{2}\left(1.35 \mathrm{~mol} \mathrm{~kg}^{-1}\right)$ at varying $R H$, which was changed from $0 \%$ to $91 \%$ and back to $0 \%$.

middle- $R H$ regions are attracted mainly by the amide groups of PVP and are less coordinated to the Co ion.

This idea is supported by the water-vapor sorption isotherm. Figure 5, obtained by replotting the data in the previous report, ${ }^{8}$ shows the humidity dependence of the water concentration ( $\mathrm{mol} \mathrm{kg}^{-1}$ or $\mathrm{mol} \mathrm{kg}^{-1} \mathrm{PVP}$ ) in PVP films with and without $\mathrm{CoCl}_{2}(1.35 \mathrm{~mol}$ $\mathrm{kg}^{-1}$ ). The water concentration of the film containing $1.35 \mathrm{~mol} \mathrm{~kg}^{-1}$ of $\mathrm{CoCl}_{2}$ is almost the same below $62 \% R H$, but is much higher above $62 \% R H$ than that of the original PVP film. It has been reported that an ethylene-vinyl alcohol copolymer containing sodium chloride sorbs water vapor more and faster than the copolymer without salt. ${ }^{13}$ In contrast, the water-vapor sorption of the PVP film is enhanced by $\mathrm{CoCl}_{2}$ only at higher $R H$ s than $62 \% R H$. The ability of $\mathrm{CoCl}_{2}$ to make the film hygroscopic is depressed in the low- and middle- $R H$ range, because Co complexes are shielded by polymer chains and can not easily interact with the adsorbed water.

Hysteresis appears to some extent in the curves of absorbance $v s$. $R H$ for the PVP films in Figure 3. The curves in the desiccation are slightly lower below $63 \% R H$ but slightly higher above $63 \% R H$ than the curves in the 

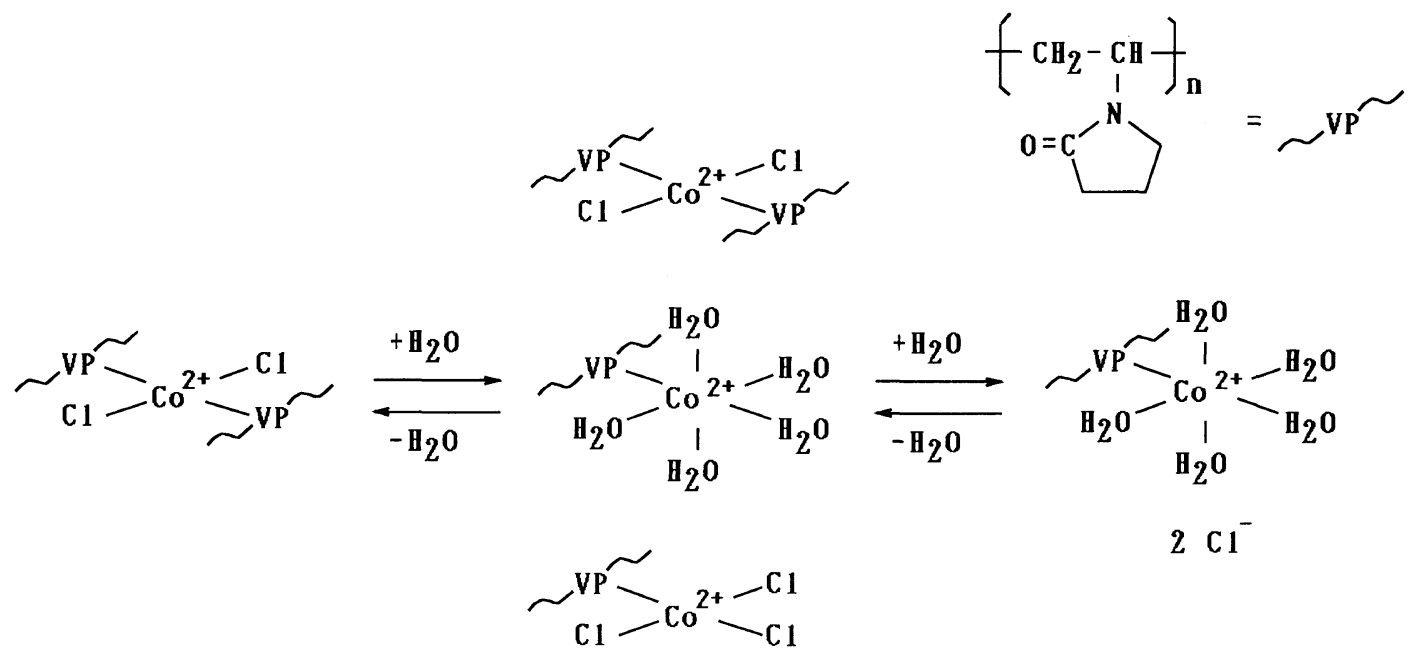

$2 \mathrm{Cl}^{-}$

(a)

(b)

(c)

Figure 6. Hydration of a PVP- $\mathrm{CoCl}_{2}$ film.

humidification. Because absorbance change arises from change in water concentration of the film, it is necessary to compare the hysteresis of absorbance with that of water concentration. The water concentration for adsorption is almost the same above $62 \% R H$ and a little lower below $62 \% R H$ than that for desorption, as can be seen in Figure 5. This hysteresis is compatible with the well known fact that a specimen, which has had greater content of water, adsorbs more water vapor at equilibrium. Hysteresis behavior in the absorbance $v s$. $R H$ curve is quite different from the case of water vapor sporption. Consequently, the abnormal hysteresis of absorbance is not due to anomalies in water vapor sorption. Because sufficiently long time was taken to record the absorbance at all data points, the difference of absorbance between the adsorption and desorption curves is that between the equilibrated values of absorbance. Therefore, absorbance is larger above $63 \% R H$ in desiccation than predicted from the corresponding water concentration. Thus, it is suggested that Co complexes interact more intimately with the medium and more easily remove water molecules during desiccation than during humidification. In other words, the average number of water molecules ligating to the Co complex is a little less during desiccation than during humidification.

\section{Hydration of a PVP-CoCl 2 Film}

It is expected that changes in coordination of the Co ion take place in the PVP films similarly to those in the MP solutions. A plausible process of hydration of the PVP film is illustrated in Figure 6. In dry films (a), almost all Co complexes probably assume the $\mathrm{CoX}_{2} \mathrm{Cl}_{2}$ form, where $\mathrm{X}$ is the carbonyl group of PVP. The coordination of two carbonyl groups of PVP to the Co ion makes a crosslinkage of the polymer in the dry state. When the water concentration is increased, the carbonyl groups of PVP are replaced by water molecules, accompanying alteration of the Co complex from the 4-coordinate to the 6coordinate (b). As a result, $\mathrm{CoX}\left(\mathrm{H}_{2} \mathrm{O}\right)_{5}{ }^{2+}$ increases instead of the $\mathrm{CoX}_{2} \mathrm{Cl}_{2}$ complex, causing the overall decrease in absorbance in the range from about 550 to $720 \mathrm{~nm}$. $\mathrm{CoXCl}_{3}{ }^{-}$ is produced by attack of the liberated chloride 
ion against the $\mathrm{CoX}_{2} \mathrm{Cl}_{2}$ complex, which results in the red shift of the absorption maximum. The formation of $\mathrm{CoXCl}_{3}{ }^{-}$is conceivable because this ionic entity may be more stable in a polar medium than a neutral one, $\mathrm{CoX}_{2} \mathrm{Cl}_{2}$; the polarity of the film is gradually increased by adsorbed water. The participation of $\mathrm{CoXCl}_{3}{ }^{-}$was proposed also in the hydration of gelatin films containing $\mathrm{CoCl}_{2} .{ }^{9}$ In fully hydrated films (c), most complexes are still linked to at least one carbonyl group, as concluded previously. ${ }^{8}$ The basis for this conclusion is the observation in the IR spectrum of a PVP film containing $\mathrm{CoCl}_{2}$. The amide $\mathrm{I}$ band is split into two peaks at 1678 and $1636 \mathrm{~cm}^{-1}$ in the dry film due to coordination of some carbonyl groups. The two peaks are put together to produce a broad peak at $1655 \mathrm{~cm}^{-1}$ when the film is hydrated, indicating that many carbonyl groups are coordinated to the complex.

\section{REFERENCES}

1. W. R. Seitz, Anal. Chem., 56, 16A (1984).

2. W. E. Morf, K. Seiler, B. Lehmann, Ch. Behringer, K. Hartman, and W. Simon, Pure Appl. Chem., 61, 1613 (1989).

3. A. P. Russell and K. S. Fletcher, Anal. Chim. Acta, 170, 209 (1985).

4. D. S. Ballantine and H. Wohtjen, Anal. Chem., 58, 2883 (1986).

5. G. Xin and H. Shanglian, Proceeding of SPIE, 1169 Fiber Optic and Laser Sensors VII, 1989, p 582.

6. F. Boltinghouse and K. Abel, Anal. Chem., 61, 1863 (1989).

7. H. Yashima, N. Miyamoto, R. Endo, and S. Furuya, Kobunshi Ronbunshu, 46, 577 (1989).

8. S. Otsuki and K. Adachi, Kobunshi Ronbunshu, 49, 697 (1992).

9. S. Otsuki and K. Adachi, J. Appl. Polym. Sci., in press.

10. S. K. Madan and J. A. Sturr, J. Inorg. Nucl. Chem., 29, 1669 (1967).

11. H. G. Biedermann and W. Graf, Z. Naturforsch. $b$, 29, 65 (1974).

12. L. I. Katzin and E. Gebert, J. Am. Chem. Soc., 72, 5464 (1950).

13. A. Apicella and H. B. Hopfenberg, J. Appl. Polym. Sci., 27, 1139 (1982). 